\title{
Sleeping Habits Predict the Magnitude of Fat Loss in Adults Exposed to Moderate Caloric Restriction
}

\author{
Jean-Philippe Chaputa Angelo Tremblay ${ }^{\mathrm{b}}$ \\ ${ }^{a}$ Children's Hospital of Eastern Ontario Research Institute, Ottawa, ON, b Department of \\ Kinesiology, Faculty of Medicine, Laval University, Quebec City, QC, Canada
}

\section{Key Words}

Adiposity $\cdot$ Diet $\cdot$ Obesity $\cdot$ Sleep $\cdot$ Weight loss

\begin{abstract}
Objective: To verify whether sleep quantity and quality at baseline predict the magnitude of fat loss in adults subjected to moderate caloric restriction. Methods: A total of 123 overweight and obese men and women (age, $41.1 \pm 6.0$ years; BMI, $33.2 \pm 3.6 \mathrm{~kg} / \mathrm{m}^{2}$ (mean \pm SD)) underwent a weight loss intervention consisting of a targeted $600-700 \mathrm{kcal} /$ day decrease in energy intake supervised by a dietician. The length of the intervention varied between 15 and 24 weeks. Body fat mass (dual-energy X-ray absorptiometry), sleep quality (total Pittsburgh Sleep Quality Index score) and sleep duration (h/night, self-reported from the Pittsburgh Sleep Quality Index) were assessed at both baseline and at the end of the weight loss program. Results: The mean weight loss over the dietary intervention was $4.5 \pm 3.9 \mathrm{~kg}, 76 \%$ of which came from fat stores. Using a multiple linear regression analysis, we observed a significant positive relationship between sleep duration and the loss of body fat, both in absolute (adjusted $\beta=0.72 \mathrm{~kg} / \mathrm{h} ; \mathrm{p}<0.05$ ) as well as in relative terms (adjusted $\beta=0.77 \% / \mathrm{h} ; \mathrm{p}<0.01$ ), after adjusting for age, sex, baseline BMI, length of the intervention, and change in total energy intake. Furthermore, we observed that a better sleep quality at baseline was associated with greater fat mass loss. Conclusion: This study provides evidence that sleeping habits can influence the success of a weight loss intervention and should be taken into consideration when one decides to start a diet.




\section{Introduction}

An accumulating body of evidence associates short sleep duration with obesity and weight gain [1]. Possible explanations include an up-regulation of appetite-stimulating hormones, a longer exposure to the obesogenic environment as well as a decrease in spontaneous physical activity [2]. Furthermore, it has recently been shown that shifting sleep duration from a short ( $\leq 6 \mathrm{~h} /$ day) to a healthier length (7-8 h/day) is associated with an attenuation of fat mass gain in adults [3]. Given that chronic sleep restriction is a common feature of our modern lifestyle, studies aimed at investigating the link between short sleep duration and obesity are relevant from a public health standpoint.

Energy restriction is widely used by obese individuals as a weight loss strategy in order to improve their metabolic profile. However, prescribing dietary interventions to obese, short-duration sleepers might be counterproductive as most of them already have higher hunger levels [4]. Moreover, lack of sleep has been reported to decrease plasma leptin levels, increase plasma ghrelin and cortisol levels, alter glucose homeostasis, and activate the orexin system, all of which impact the control of appetite and might compromise the efficacy of dietary interventions [5]. Interestingly, Nedeltcheva et al. [6] recently reported that insufficient sleep undermines dietary efforts to reduce adiposity. In their crossover study, 10 overweight adults were randomly assigned to sleep either $5.5 \mathrm{~h}$ or $8.5 \mathrm{~h}$ each night for 14 days in conjunction with moderate caloric restriction in a closed clinical research environment. Compared with participants who slept $8.5 \mathrm{~h}$ per night, participants who slept only $5.5 \mathrm{~h}$ lost less body fat and more fat-free body mass, and had less favorable changes in metabolic hormones and in substrate and energy utilization. In addition, participants in the shorter sleep group perceived greater hunger than participants in the longer sleep group. These findings are novel and suggest that adequate sleep might be an important factor in successful weight loss; however, these results need to be replicated in a real life setting, because the tightly controlled sleep laboratory setting does not provide information about other environmental factors that might influence compliance to weight-reducing diets.

The objective of the present study is to verify whether sleeping habits affect the magnitude offatloss in overweight and obese adults subjected to moderate caloric restriction. We hypothesize that both sleep quantity and quality at baseline predict the success of a weight-reducing program in adults.

\section{Subjects and Methods}

\section{Subjects}

In order to maximize power, three weight loss studies performed in our facilities (Laval University, Quebec, Canada) have been pooled together. Details on these studies have been published elsewhere [7-9]. Briefly, these weight-reducing interventions aimed at verifying the effect of either calcium + vitamin D supplementation, milk supplementation or a functional diet on body fat loss and various indicators of health. Only individuals involved in the control groups of these studies have been kept for statistical analyses in order to avoid the influence of the active ingredient on the main outcome measure (i.e. fat loss). Briefly, healthy overweight and obese men and women aged between 25 and 50 years were subjected to a weight loss intervention consisting of a targeted 600-700 kcal/day decrease in energy intake supervised by a dietician. The following inclusion criteria were also considered: absence of menopause (determined by the cessation of menstruation), stable body weight (body weight change $<3 \mathrm{~kg}$ for 2 months before intervention), $<3$ periods of 20 min of physical activity per week, no use of medication that could affect body weight, no smoking, normal blood pressure values $(<140 / 90 \mathrm{~mm} \mathrm{Hg}$ ), consumption of $\leq 10$ alcoholic beverages per week, and consumption of $\leq 5$ cups of coffee per day. The length of the intervention varied between 15 and 24 weeks, depending on the study. Each participant met his/her assigned dietician every 2 weeks until the completion of the study. Compliance was assessed by comparing the diet prescribed 
Chaput et al.: Sleeping Habits Predict the Magnitude of Fat Loss in Adults Exposed to Moderate Caloric Restriction

(total daily energy intake and macronutrient composition) to the actual diet composition of the participants, the latter assessed every 2 weeks by means of $24 \mathrm{~h}$ food recalls. Study protocols were approved by the Laval University Ethics Committee. Written informed consent was obtained from all participants.

\section{Anthropometric and Body Composition Measurements}

Height was measured to the nearest $0.1 \mathrm{~cm}$ using a standard stadiometer, and body weight was measured to the nearest $0.1 \mathrm{~kg}$ using a digital panel indicator scale (Beckman Industrial, Glenrothes, UK). BMI was calculated as body weight divided by height squared $\left(\mathrm{kg} / \mathrm{m}^{2}\right)$. Waist circumference was measured at the line between the lower border of the last rib and the upper border of the iliac crest. Body fat mass was measured by dual-energy X-ray absorptiometry (GE Medical Systems Lunar, Diegem, Belgium). All measurements were performed according to standardized procedures at both baseline and at the end of the weight loss program.

\section{Sleep Assessment}

At baseline, each participant completed the Pittsburgh Sleep Quality Index (PSQI), a self-rated questionnaire that assesses sleep quality and disturbances over the preceding 1-month time interval [10]. Briefly, 19 individual items generate seven component scores: subjective sleep quality, sleep latency, sleep duration, habitual sleep efficiency, sleep disturbances, use of sleeping medication, and day time dysfunction. The sum of the component scores yields one total score with a maximum of 21. A total PSQI score greater than 5 is highly sensitive and specific in distinguishing good from poor sleepers [10] and has been validated in a number of populations [11]. For the purpose of this study, both sleep quality (total PSQI score) and sleep duration (self-reported from the PSQI) were used for statistical analyses.

\section{Diet Assessment}

Diet was evaluated with a 3-day food record, including 2 weekdays and 1 weekend day, at baseline and at the end of the intervention. Participants were shown how to complete the record by a dietician who provided instructions about measuring the quantities of ingested foods. This method of dietary assessment has been shown to provide a relatively reliable measure of diet in this population [12]. Mean daily energy intake was estimated by a dietician using a computerized version of the Canadian Nutrient File [13]. The change in total energy intake from baseline (in $\mathrm{kJ} /$ day) was used in the analyses as a covariate.

\section{Statistical Analysis}

Since there was no statistically significant sex interaction between sleep duration and the outcome variable, data for both sexes were combined to improve clarity and maximize power. Sleep quality and quantity at baseline were used to predict the magnitude of fat loss using multiple linear regression analysis. The model was adjusted for age, sex, baseline BMI, length of the intervention, and change in energy intake as covariates. A two-tailed $p$ value of less than 0.05 was considered to indicate statistical significance. All statistical analyses were performed using the JMP version 8 program (SAS Institute, Cary, NC, USA).

\section{Results}

Baseline characteristics of participants are shown in table 1 . The mean weight loss of all participants over the dietary intervention was $4.5 \pm 3.9 \mathrm{~kg}$ (mean \pm SD), $76 \%$ of which came from fat stores. The 24-hour food recalls showed that participants complied at $\geq 90 \%$ with their energy-restricted diet, as assessed by the difference between total energy intake actually consumed throughout the program and the prescription.

After adjusting for age, sex, baseline BMI, length of the intervention, and change in total energy intake, we observed a significant positive relationship between sleep duration and the loss of body fat, both in absolute (adjusted $\beta=0.72 \mathrm{~kg} / \mathrm{h} ; \mathrm{p}<0.05$ ) as well as in relative terms (adjusted $\beta=0.77 \% / h ; p<0.01$ ) (fig. 1A, 1B). Furthermore, sleep quality as assessed by the PSQI was inversely related to both fat mass loss (adjusted $\beta=-0.19 ; \mathrm{p}<0.05$ ) and percent body fat loss (adjusted $\beta=-0.21$; $<<0.01$ ) after adjusting for covariates (fig. 1C, 1D), 


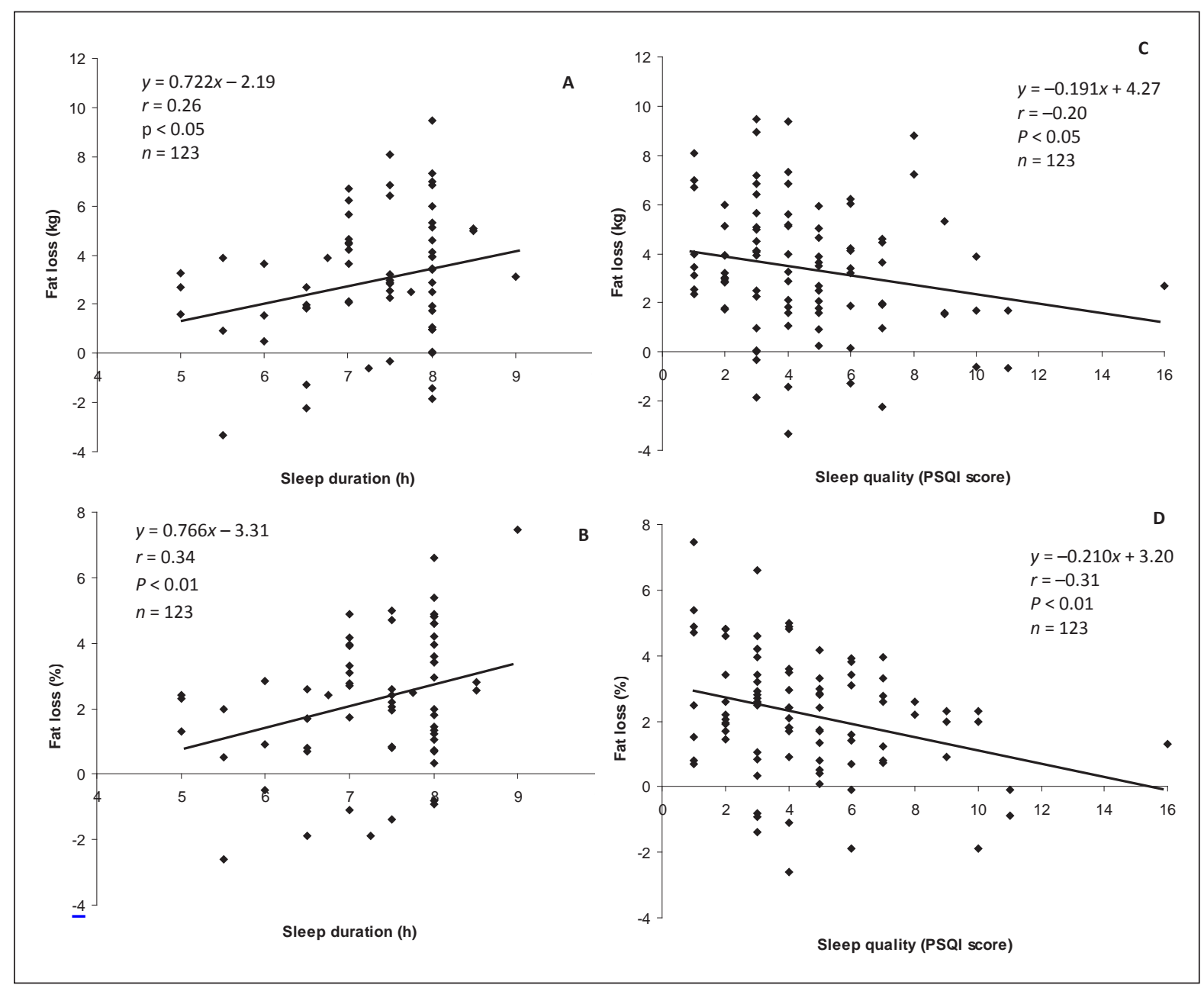

Fig. 1. Sleep duration in relation to $\mathbf{A}$ absolute body fat loss and $\mathbf{B}$ percent body fat loss in adults after adjusting for age, sex, baseline BMI and the change in total energy intake. Sleep quality in relation to $\mathbf{C}$ absolute body fat loss and $\mathbf{D}$ percent body fat loss in adults after adjusting for age, sex, baseline BMI and the change in total energy intake. PSQI = Pittsburgh Sleep Quality Index.

suggesting that a better sleep quality was associated with greater fat mass loss. Of note, removing the outlier (PSQI score of 16) from the analyses did not significantly change our results (data not shown).

\section{Discussion}

Collectively, we observed that both total sleep time (h/night) and sleep quality (PSQI score) predict fat mass loss during typical dietary interventions in overweight and obese adults. Specifically, an increase by $1 \mathrm{~h}$ in sleep duration was associated with a decrease of $0.7 \mathrm{~kg}$ in fat mass after adjustment for covariates. Our study suggests that sleeping habits can affect the success of a weight loss intervention and should be taken into account when one decides to start a diet. Future studies should put efforts in elucidating the mechanisms behind this observation. 
Chaput et al.: Sleeping Habits Predict the Magnitude of Fat Loss in Adults Exposed to Moderate Caloric Restriction

Table 1. Baseline characteristics of participants $(\mathrm{n}=123)^{\mathrm{a}}$

\begin{tabular}{ll}
\hline Age, years & $41.1 \pm 6.0$ \\
Sex (n) & 70 \\
$\quad$ Men & 53 \\
$\quad$ Women & $94.7 \pm 14.5$ \\
Body weight, kg & $33.2 \pm 3.6$ \\
BMI, kg/m² & $106.4 \pm 10.1$ \\
Waist circumference, cm & $35.7 \pm 8.3$ \\
Fat mass, kg & $38.1 \pm 7.1$ \\
Fat mass, $\%$ & $7.1 \pm 1.0$ \\
Sleep duration, h & $4.8 \pm 2.9$ \\
PSQI (total score) & $5,242 \pm 2031$ \\
Energy intake, kJ/day & \\
\hline \multicolumn{2}{c}{ PSQI = Pittsburgh Sleep Quality Index. } \\
$\quad$ aValues are mean 8 SD or n.
\end{tabular}

Our results agree with a recent study showing that sleep time predicted success in a weight loss program consisting of an intensive 6-month behavioral weight loss intervention in 472 obese adult participants [14]. The intervention consisted of 22 group sessions led by behavioral counselors, and recommendations included reducing dietary intake by 500 kcal/day, adopting the Dietary Approaches to Stop Hypertension (DASH) dietary pattern, and increasing physical activity to at least $180 \mathrm{~min} /$ week. Specifically, the authors found that participants sleeping $\leq 6 \mathrm{~h}$ or $\geq 8 \mathrm{~h}$ daily were less likely to achieve eligibility for phase II (i.e. the randomized weight loss maintenance portion of the study) than those sleeping $>6$ and $\leq 8 \mathrm{~h}$ a day. Thus, these observations further emphasize the need to consider 'sleep' as part of any weight loss intervention.

In the context of weight loss, dietary restriction is widely used as a means of inducing a caloric deficit. Recent results showed that sleep restriction attenuates the effect of a reducedcalorie diet on excess adiposity [6]. Given that higher ghrelin concentrations may facilitate the retention of fat $[15,16]$ and increased hunger could compromise adherence to caloric restriction, these mechanisms may help explain why lack of sufficient sleep may compromise the efficacy of commonly used dietary interventions. Future studies will be instrumental in determining the longer-term effects of reduced sleep on body composition, energy metabolism, and substrate utilization in weight-reduced individuals.

The present study has some limitations that need to be taken into consideration before generalizing our study findings. Firstly, the small sample size and preliminary nature of this investigation precludes any definitive conclusion. However, we hope that it will generate larger and well-designed studies to address this issue in a timely fashion. Furthermore, we have to keep in mind the well-known limitations of questionnaire-based measurements (e.g. sleep duration and diet assessment) as well as the possibility of residual confounding. Other factors (e.g. personality traits, educational factors, or other lifestyle qualities) may affect sleeping habits as well as adherence to a dietary intervention and might then weaken the observed associations. Finally, the present study is well suited for the identification of associations and does not permit causal inferences.

In summary, our study provides evidence to the effect that both sleep quantity and quality at baseline can predict the magnitude of fat loss in adults exposed to moderate caloric restriction. These results suggest that adequate sleep might influence the success of a weight loss intervention, and perhaps sleep should be included as part of the lifestyle package that traditionally has focused on diet and exercise. 


\section{Acknowledgements}

We express our gratitude to the subjects for their participation and the staff of the Physical Activity Sciences Laboratory at Laval University for their contribution to this study. None of the authors had a personal interest or potential conflict of interest with the organizations sponsoring the projects. Funding to conduct these studies was obtained from Wyeth Consumer Healthcare Inc., the Dairy Farmers of Canada, the US National Dairy Council and the Canadian Institutes of Health Research. JPC holds a Junior Research Chair in Healthy Active Living and Obesity Research. AT is partly funded by the Canada Research Chair in Environment and Energy Balance.

\section{Disclosure Statement}

The authors declare no conflict of interest.

\section{References}

1 Nielsen LS, Danielsen KV, Sørensen TI: Short sleep duration as a possible cause of obesity: critical analysis of the epidemiological evidence. Obes Rev 2011;12:78-92.

- 2 Chaput JP, Klingenberg L, Sjödin AM: Do all sedentary activities lead to weight gain: sleep does not. Curr Opin Clin Nutr Metab Care 2010;13:601-607.

- 3 Chaput JP, Després JP, Bouchard C, Tremblay A: Longer sleep duration associates with lower adiposity gain in adult short sleepers. Int J Obes (Lond) 2012;36:752-756.

- 4 Spiegel K, Tasali E, Penev P, Van Cauter E: Brief communication: Sleep curtailment in healthy young men is associated with decreased leptin levels, elevated ghrelin levels, and increased hunger and appetite. Ann Intern Med 2004;141:846-850.

- 5 Leproult R, Van Cauter E: Role of sleep and sleep loss in hormonal release and metabolism. Endocr Dev 2010;17:11-21.

- 6 Nedeltcheva AV, Kilkus JM, Imperial J, Schoeller DA, Penev PD: Insufficient sleep undermines dietary efforts to reduce adiposity. Ann Intern Med 2010;153:435-441.

7 Major GC, Alarie F, Doré J, Phouttama S, Tremblay A: Supplementation with calcium + vitamin D enhances the beneficial effect of weight loss on plasma lipid and lipoprotein concentrations. Am J Clin Nutr 2007;85: 54-59.

- 8 Gilbert JA, Joanisse DR, Chaput JP, Miegueu P, Cianflone K, Alméras N, Tremblay A: Milk supplementation facilitates appetite control in obese women during weight loss: a randomized, single-blind, placebocontrolled trial. Br J Nutr 2011;105:133-143.

- 9 Drapeau V, Arguin H, Tremblay A: Impact of a satiating diet on satiety quotient and body weight in obese men. Appl Physiol Nutr Metab 2010;35:373 (abstract).

10 Buysse DJ, Reynolds CF, Monk TH, Berman SR, Kupfer DJ: The Pittsburgh Sleep Quality Index: a new instrument for psychiatric practice and research. Psychiatry Res 1989;28:193-213.

-11 Carpenter JS, Andrykowski MA: Psychometric evaluation of the Pittsburgh Sleep Quality Index.J Psychosom Res 1998;45:5-13.

12 Tremblay A, Sévigny J, Leblanc C, Bouchard C: The reproducibility of a three-day dietary record. Nutr Res 1983;3:819-830.

13 Government of Canada: The Canadian Nutrient File. Ottawa, Canada, Health and Welfare Canada, 2005.

-14 Elder CR, Gullion CM, Funk KL, DeBar LL, Lindberg NM, Stevens VJ: Impact of sleep, screen time, depression and stress on weight change in the intensive weight loss phase of the LIFE study. Int J Obes (Lond) 2012; 36:86-92.

15 Rodríguez A, Gómez-Ambrosi J, Catalán V, Gil MJ, Becerril S, Sáinz N, Silva C, Salvador J, Colina I, Frühbeck G: Acylated and desacyl ghrelin stimulate lipid accumulation in human visceral adipocytes. Int J Obes (Lond) 2009;33:541-552.

-16 Tschöp M, Smiley DL, Heiman ML: Ghrelin induces adiposity in rodents. Nature 2000;407:908-913. 